\title{
Öffentliche Aufrufe für eine verfassunggebende Versammlung
}

Der Weg zur Vereinigung der beiden deutschen Staaten führt, so belehrt uns die herrschende Meinung in Politik und Staatslehre, über den Beitritt gemäß Art. ${ }_{3} 3$ S. 2 GG. Gleich hundert Lehrer des Staatsrechts an deutschen Universitäten umstellten mit einer öffentlichen Resolution schützend diesen Artikel, obwohl dieser angesichts der obwaltenden Mehrheitsverhältnisse kaum schutzbedürftig war. Wir dokumentieren im folgenden eine andere Auffassung, die davon ausgeht, daß die Neugründung eines politischen Gemeinwesens auf der Tagesordnung stand (und steht), die der historischen Situation angemessen und Art. 146 GG entsprechend nur in einer öffentlichen Debatte und einer Volksabstimmung über die gemeinsame Verfassung zum Ausdruck kommt.

\section{A. Wo bleibt das Volk? - Plädoyer für eine verfassunggebende Versammlung}

Nachdem im Herbst 1989 oppositionelle Gruppen in der DDR eine durch Zivilcourage und Gewaltlosigkeit beeindruckende demokratische Revolution ins Werk gesetzt haben, scheinen führende westdeutsche Politiker nun den elektrisierenden Ruf »Wir sind das Volk « durch eine überstürzte Einigung neutralisieren zu wollen. Wahltaktisch motiviert und mit einem alles verdrängenden Interesse an der Wahrung ihrer politischen Besitzstände, legen sie ihren Parteien wie auch den »Schwesterparteien « der DDR nahe, für die Einigung den Beitritt nach Artikel 23 des Grundgesetzes zu wählen. Die zu bildenden Länder der DDR oder auch nur deren neu gewählte Volkskammer sollen diesen Beitritt erklären können. Von dem Volk, das eben noch ein totalitäres Regime stürzte, und auch von jenem Volk, dem nach der Präambel des Grundgesetzes aufgegeben ist, »in freier Selbstbestimmung die Einheit und Freiheit Deutschlands zu vollenden «, ist nicht mehr die Rede. Daß das Grundgesetz bis zu diesem Zeitpunkt als Übergangsverfassung gedacht war, soll nun nicht mehr gelten.

Wir sind mit der Verheißung des Grundgesetzes aufgewachsen, über ein gemeinsame Verfassung für alle Deutschen und über die Gestalt unseres politischen Gemeinwesens selbst bestimmen zu können, wenn der Weg dazu frei ist. Deshalb plädieren wir dafür, den vom Grundgesetz selbst in seiner Präambel und im Artikel I 46 vorgezeichneten Pfad zu einer neuen gemeinsamen Verfassung zu gehen und über eine verfassungsgebende Versammlung

- sich das Bewährte in öffentlicher Debatte bewähren zu lassen,

- den Menschen in beiden Teilen Deutschlands die historische Chance zu eröffnen, ihre Vorstellungen von Einheit und von verfassungsförmiger Gemeinsamkeit zur Geltung zu bringen. 
- die Selbstachtung und Würde der Bürger und Bürgerinnen der DDR zu wahren, die sich unter erheblichen Risiken ihre politische Freiheit erkämpft haben.

Dieser Aufruf wurde von folgenden Wissenschaftlern und Wissenschaftlerinnen unterstützt:

Erhard Denninger (Frankfurt/Main), Michael Stolleis (Frankfurt/Main), Theodor Schweısfurth (Heidelberg), Jürgen Habermas (Frankfurt/Main), Ernst Tugendhat (Berlin), Michael Theunissen (Berlin), Marianne Rodenstein (Frankfurt/Main), Axel Honneth (Berlin), Friedrich Kramer (Berlin), Cornelius Prittwitz (Frankfurt/Main), Klaus Günther (Frankfurt/Main), Irma Hanke (München), Dieter Sterzel (Oldenburg), Rudolf Wiethölter, (Frankfurt/Maın), Adrienne Göhler (Hamburg), Ilse Staff (Frankfurt/Maın), Lutz Niethammer (Essen), Claus Offe (Bremen), Oskar Negt (Hannover), Ulrich K. Preuß (Berlin), Gerd Winter (Bremen), Dian Schefold (Bremen), Manfred Weiss (Frankfurt/Main), Ute Gerhard (Bremen/Frankfurt), Hans Burgbacher (Hamburg), Diemut Majer (Karlsruhe/Bern), Rainer Schröder (Hannover), Hubert Rottleuthner (Berlin), Uwe Wesel (Berlin), Erhard Blankenburg (Amsterdam), Wolfgang Däubler (Bremen), Gert Brüggemeier (Bremen), Rolf Knieper (Bremen), Johannes Feest (Bremen), Oskar Hartwig (Hannover), Joachim Perels (Hannover), Thomas Blanke (Oldenburg), Alexander Roßnagel (Heidelberg), Peter Derleder (Bremen), Helmut Kohl (Frankfurt/ Main), Ludwig Salgo (Esslingen), Volkmar Gessner (Bremen), Lorenz Bölinger (Bremen), Robert Francke (Bremen), Dieter Hart (Bremen), Wolfgang van den Daele (Berlin), Ulrıch Stascheit (Frankfurt/Main), Bernd Schulte (München), Berthold Huber (Frankfurt/Maın), Raıner Wolf (Düsseldorf), Monika Frommel (Frankfurt/Maın), Andreas Zielcke (München), Klaus Sieveking (Bremen), Konstanze Plett (Bremen), Hans Nichlitz (Bremen), Harald Beutlage (Bremen), Margaretha Sudhof (Frankfurt/Main), Cornelius Nestler-Tremel (Frankfurt/Main), Wolfgang Kohl (Frankfurt/Main), Felix Herzog (Frankfurt/Main), Rainer Frey (Frankfurt/Main), Stephan Meder (Frankfurt/Main), Rainer Erd (Frankfurt/Main), Rainer Keller (Hannover), Hans Joas (Berlin), Peter Grottian (Berlin), Michael Greven (Marburg), Claus Leggewıe (Gießen), Eberhard Schmidt (Oldenburg), Barbara Knieper (Bremen), Mechthild Rumpf (Hannover), Jürgen Seifert (Hannover), Yvonne Schütze (Berlin), Richard van Dülmen (Saarbrücken), Karl F. Schumann (Bremen), Margrit Brückner (Frankfurt/Main), K. Lorenz (Saarbrücken), Bernd Ostendorf (München), Rudolf Billerbeck (Bremen), Birgit Meyer (Esslingen), Helmut Wiesenthal (Bremen), Gottfried Erb (Giessen), Michael Vester (Hannover), Ilse Dröge-Modelmog (Oldenburg), Erhard Lucas-Busemann (Oldenburg), KJaus W. Schüler (Oldenburg), Stefan Müller-Doohm (Oldenburg), Siegfried Grubitzsch (Oldenburg), Hans Uszkorett (Saarbrücken), H. J. Neuschäfer (Saarbrücken), Jochen Schlobach (Saarbrücken), Alfred Sander (Saarbrücken), Wolfgang Buchholz (München), Johannes Ingelkamp (Saarbrücken), Christoph Sachße (Kassel), Rainer Rath (Saarbrücken), Rainer Tetzlaff (Hamburg), Wilhelm Schumm (Frankfurt/Main), Lutz-Rainer Reuter (Hamburg), Volker Sigusch (Frankfurt/Main), Jürgen Fijalkowski (Berlin), Helga Neubeck-Fischer (München), Gerhard Stuby (Bremen), Rainer Dilcher (Frankfurt/Main), Gerhard Kraiker (Oldenburg), Hubert Lehmeier (Frankfurt/Main), Peter Hanke (München), Klaus Herding (Hamburg), Horst Bredekamp (Hamburg), Bruno Reudenbach (Hamburg), Carles Ossorio (Darmstadt), Elmar Altvater (Berlin), Waldemar Schulte (Frankfurt/Main), Sabine Rothe (Frankfurt/ Main), Monika Steinhauser (Berlin), Otmar Jung (Berlin), Bernd Greiner (Hamburg), Christine Zeile (München), Wolfgang Bonss (Hamburg), Carola Sachse (Berlin), Lerke Gravenhorst (München), Barbara Pieper (München), Karin Jurczyk (München), Ina Fuchs (München), Rudolf Pettınger (Schöngeising), Herbert Blümel (München), Konrad Leube (Sulzbg./ Allgäu), Grete Tüllmann (München), Charlotte Schach-Glass (Hamburg), Monika Wagner (Hamburg), Carmen Tatschmurat (München), Hans-Rudolf Leu (München), Yolanda M. Koller-Tejero (München), Heidrun Hoppe (München), Tilla Siegel (Frankfurt/Main), Peter Noller (Frankfurt/Main), Rainer Deppe (Frankfurt/Maın), Andrea Maihofer (Frankfurt/ Maın), Rainer Döbert (Berlin), Ingeborg Maus (Frankfurt/Main), Reimut Reıche (Frankfurt/ Main), Alex Demirovic (Frankfurt/Main), Sigrid Meuschel (Berlin), Ulrich Rödel (Frankfurt/ Main), Helmut Dubiel (Frankfurt/Main), Christel Eckart (Hamburg), Albrecht Funk (Berlin), Birgit Geissler (Bremen), Günter Warsewa (Bremen), Karl-Werner Brand (München), Meinhard Sander (München), Richard Pieper (München), Hans-Georg Isenburg (Bremen), HansJoachım v. Kondratowitz (Berlin), Klaus Neubeck (München), Eberhard K. Seifert (Hamburg), Regıne Kollek (Hamburg), Paula Bradisch (Hamburg), Ina Güse (Bremen), Barbara Loer (Bremen), Michael Gagern (München), Wolfgang Littek (Bremen), Bodo Vorgt (Bremen), Detlev Gronwald (Bremen), Willi Bruns (Bremen), Klaus Grimmer (Kassel), Heribert Schatz (Duisburg), Margrit Grubmüller (Würzburg), Wolfgang Nitsch (Oldenburg), Irene Berger (München), Susanne Pelzer (München), Jens Lipsky (München), Colbey-Schrader 
(Kochel), Irene Herzberg (München), Marianne Krug (Sulzberg), Ursula Nissen (München), Alfred Hössl (Ottobrunn), Gisela Dittrich (München), Johann de Rijke (München), Heike Elskemper-Mader (München), Jutta Stıch (München), Heinz Schattner (München), Carsten Rummel (München), Elisabeth Marsch (München), Monika Jaeckel (München), Wolfgang Kraus (München), Lusse Behunger (Augsburg), Margarete Landenberger (München), M.H. Müller-Rytlewskı (Berlin), Rolf Rytlewski (Berlin), Klaus Dörner (Gütersloh), Dieter Rucht (Berlin), Jürgen Gerhards (Berlin), Barbara Blattert (Berlin), Thomas Ohlemacher (Berlin), Donatella delle Parte (Berlin), Josef Esser (Frankfurt/Main), Hans-Georg Flichinger (Kassel), Friedrich Ortmann (Kassel), Günter Frankenberg (Frankfurt/Main).

\section{B. Plädoyer für eine verfassunggebende Versammlung}

Nachdem im Herbst 1989 oppositionelle Gruppen in der DDR eine durch Zivilcourage und Gewaltlosigkeit beeindruckende demokratische Revolution ins Werk gesetzt und am 18. März 1990 die ersten freien Wahlen in der Geschichte der DDR stattgefunden haben, stellt sich jetzt die drängende Frage, wie das verfassungsrechtliche Dach eines geeinten Deutschland beschaffen sein soll. Welche Wege hierzu beschritten werden, hängt nach unserer Auffassung nicht nur von der politischen Zweckmäßigkeit oder der größeren Praktikabilität ab. Wie auch immer die Einheit erreicht werden mag - für Art. ${ }_{2}$ GG mögen in der Tat praktische Gründe sprechen - in jedem Fall sollte ein gemeinsamer Neubeginn gewagt werden.

Die dem Deutschen Volk in beiden Teilen Deutschlands sich bietende historische Chance, »in freier Selbstbestimmung die Einheit und Freiheit Deutschlands zu vollenden «, verlangt danach,

- jeden Anschein der verfassungsrechtlichen »Einvernahme» der DDR zu vermeiden und

- den Menschen in beiden Teilen Deutschlands die Gelegenheit zu eröffnen, ihre Vorstellungen von Einheit und von verfassungsförmiger Gemeinsamkeit zur Geltung zu bringen.

Eine gemeinsam erarbeitete und beschlossene Verfassung, die in weiten Teilen auf dem Bewährten des Grundgesetzes aufbauen kann, wäre ein guter und der historischen Situation angemessener Start für ein geeintes Deutschland. Daher plädieren wir dafür, nach der Herstellung der staatlichen Einheit den vom Grundgesetz selbst in seiner Präambel und in Art. ${ }_{4} 6$ vorgezeichneten Pfad zu einer neuen gemeinsamen Verfassung zu gehen, mit ihrer Ausarbeitung eine verfassunggebende Versammlung zu betrauen und damit dem geeinten Deutschland auf dem klassischen Weg der Verfassungsgebung eine bekräftigte und dadurch gestärkte Grundlage zu geben.

Hans-Herbert von Arnim (Speyer), Alexander Blankenagel (Würzburg), Michael Bothe (Frankfurt), Brun-Otto Bryde (Gießen), Hans Peter Bull (Hamburg/Kiel), Erhard Denninger (Frankfurt), Ingwer Ebsen (Münster), Helmut Goerlich (Hamburg), Wolfgang Hoffmann-Riem (Hamburg), Erich Küchenhoff (Münster), JörgDetlef Kühne (Hannover), Klaus Lange (Gießen), Hans Meyer (Frankfurt), Ingolf Pernice (Brüssel), Bernhard Schlink (Bonn), Walter Schmidt (Frankfurt), HansPeter Schneider (Hannover), Peter Schneider (Mainz), Helmuth Schultze-Fielitz (Bonn), Gunnar Folke Schuppert (Augsburg), Ilse Staff (Frankfurt), Heinhard Steiger (Gießen), Martin Stock (Bielefeld), Michael Stolleis (Frankfurt), Dieter Suhr (Augsburg), Rainer Wahl (Freiburg), Joachim Wieland (Freiburg), Friedrich von Zezschwitz (Gießen). 